\title{
Research Article \\ Effect of Dead Load on Dynamic Characteristics of Rotating Timoshenko Beams
}

\author{
Yan-Qi Yin, ${ }^{1}$ Bo Zhang, ${ }^{1}$ Yue-ming Li, ${ }^{1}$ and Wei-Zhen $L u^{2}$ \\ ${ }^{1}$ State Key Laboratory for Strength and Vibration of Mechanical Structures, School of Aerospace Engineering, Xian Jiaotong University, \\ Xian 710049, China \\ ${ }^{2}$ Department of Architecture and Civil Engineering, City University of Hong Kong, Kowloon, Hong Kong
}

Correspondence should be addressed to Yue-ming Li; liyueming@mail.xjtu.edu.cn

Received 22 May 2015; Revised 13 August 2015; Accepted 16 August 2015

Academic Editor: Paolo Maria Mariano

Copyright ( 2015 Yan-Qi Yin et al. This is an open access article distributed under the Creative Commons Attribution License, which permits unrestricted use, distribution, and reproduction in any medium, provided the original work is properly cited.

\begin{abstract}
The dynamic characteristics of a rotating cantilever Timoshenko beam under dead load are investigated in this paper. Considering the predeformation caused by dead load and centrifugal force, governing equation of rotating cantilever Timoshenko beam is derived based on Hamilton's principle, and the influence of the load on natural vibration is revealed. A suit of modal experimental apparatus for cantilever beam is designed and used to test the natural frequencies under the dead load, and the natural frequencies under rotation condition are calculated with a commercial finite element code. Both the experimental result and numerical result are utilized to compare with the present theoretical result, and the results obtained by present modeling method show a good agreement with those obtained from the experiment and finite element method. It is found that the natural frequencies of cantilever beam increase with both the dead load and the rotating speed.
\end{abstract}

\section{Introduction}

Turbine blades serve in complex environment since both high-pressure gas and high-speed rotation act on it during operating. Turbine blade can be idealized as rotating beams, and its dynamic analysis is of great importance to design such structures properly.

In terms of rotation of a turbine blade, many researches have been carried out on the basis of beam model. Yokoyama [1] derived the governing equations from Hamilton's principle for free vibrations of rotating Timoshenko beams and analyzed the characteristics with the help of finite element technique. Du et al. [2] presented an expression to solve analytically for the exact natural frequencies and modal shapes of rotating Timoshenko beams. Lin and Hsiao [3] derived governing equations for linear vibration of a rotating Timoshenko beam and proposed a method based on power series solution to solve the natural frequency. Rao and Gupta [4] derived the stiffness and mass matrices of a rotating Timoshenko beam and calculated natural frequencies using finite element method. Banerjee and Sobey [5] proposed energy expressions for rotating tapered Timoshenko beams.
Their work complemented important terms which had been omitted by some authors. Based on the previous work, Banerjee et al. [6] used the dynamic stiffness method to investigate the free bending vibration of rotating tapered beams. Ozgumus and Kaya [7] analyzed free vibration of a rotating, tapered Timoshenko beam considering flapwise bending vibration.

For the study of dead load, there are some papers available in literature which discussed the effect on Euler beams. Kelly et al. [8] presented a theoretical analysis of a vibratory beam by considering an initial deformation of the system. Takabatake [9] introduced the effect of dead load in static beams and obtained the governing equations of BernoulliEuler beam. Takabatake [10] examined the effect of dead load on the natural frequencies of simply supported or fixed beam based on the previous work through Galerkin method. He presented a closed-form approximate solution of the natural frequencies of simply supported beams and indicated that natural frequencies increased with dead load and explained the key factor governing the effect. With the similar way, Zhang and Ren [11] solved the influence of dead load on beams through a dynamic stiffness-based method. 


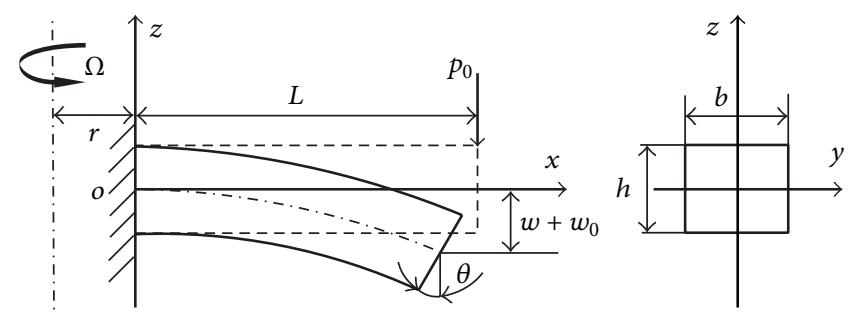

FIGURE 1: Sketch of a rotating Timoshenko beam under dead load.

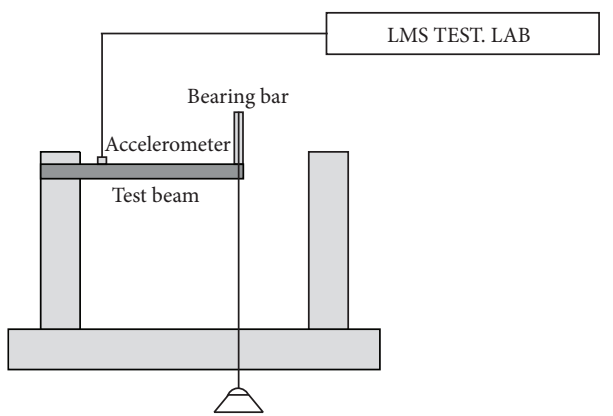

(a) Front view

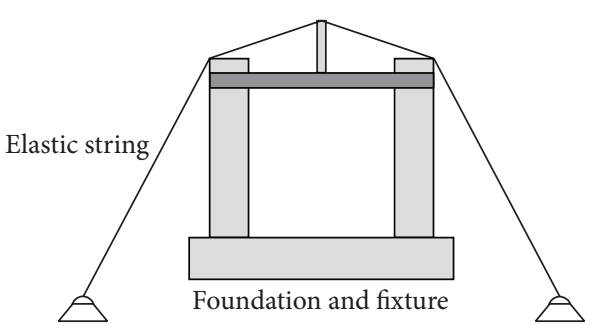

(b) Left view

FIgURE 2: Experimental setup of modal test for the beam with a dead load.

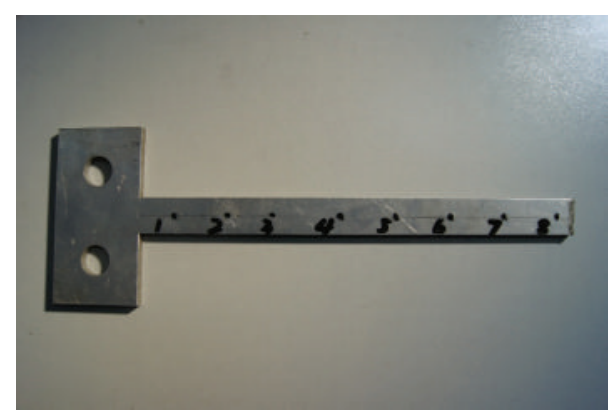

Figure 3: Test aluminum alloy beam.

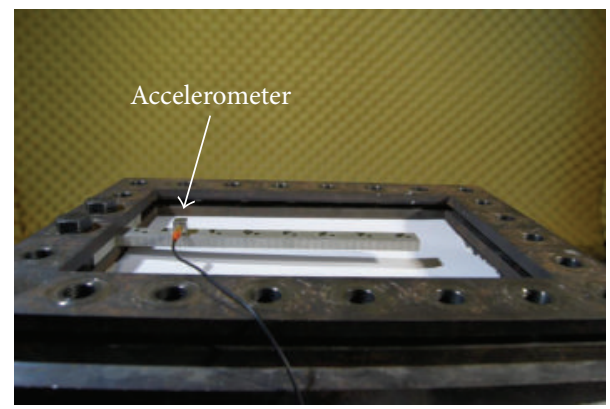

FIGURE 4: Environment of the modal test.

For complex structures, such as a cable-stayed bridge, finite element method is proposed on the basis of Takabatake's work to solve similar problems. For instance, Zhou and Zhu [12] developed a conception of load-induced stiffness matrix

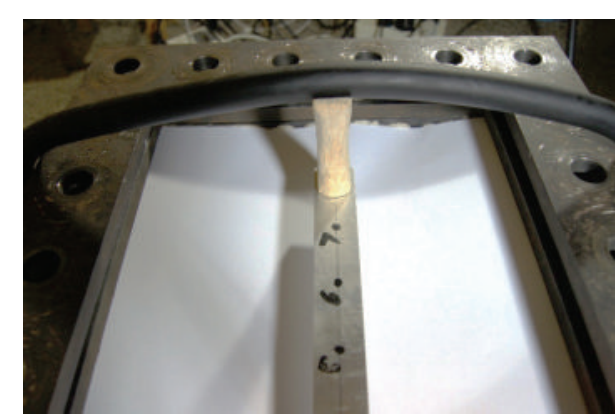

FIGURE 5: Setup diagram of applying the dead load.

and derived a finite element method according to Hamilton's principle.

In respect of rotation research, few works, however, deal with the effect of dead load on the dynamical characteristics of a rotational beam. Researches of dead load acting on beams conduct without considering the rotation effect, and moreover they focus on Euler beam without considering the effect of shear deformation and rotary inertia. In the present paper, we consider a short turbine blade as Timoshenko beam which is more suitable to model its dynamic characteristics and study the vibration characteristics of rotating Timoshenko beams with a dead load. The combination effects of centrifugal tension and lateral dead load on natural frequencies are taken into account, and variation of natural frequencies with two factors is derived. To verify the proposed theoretical model, both the modal experiment for cantilever beam under the dead load and the numerical computation for rotating 

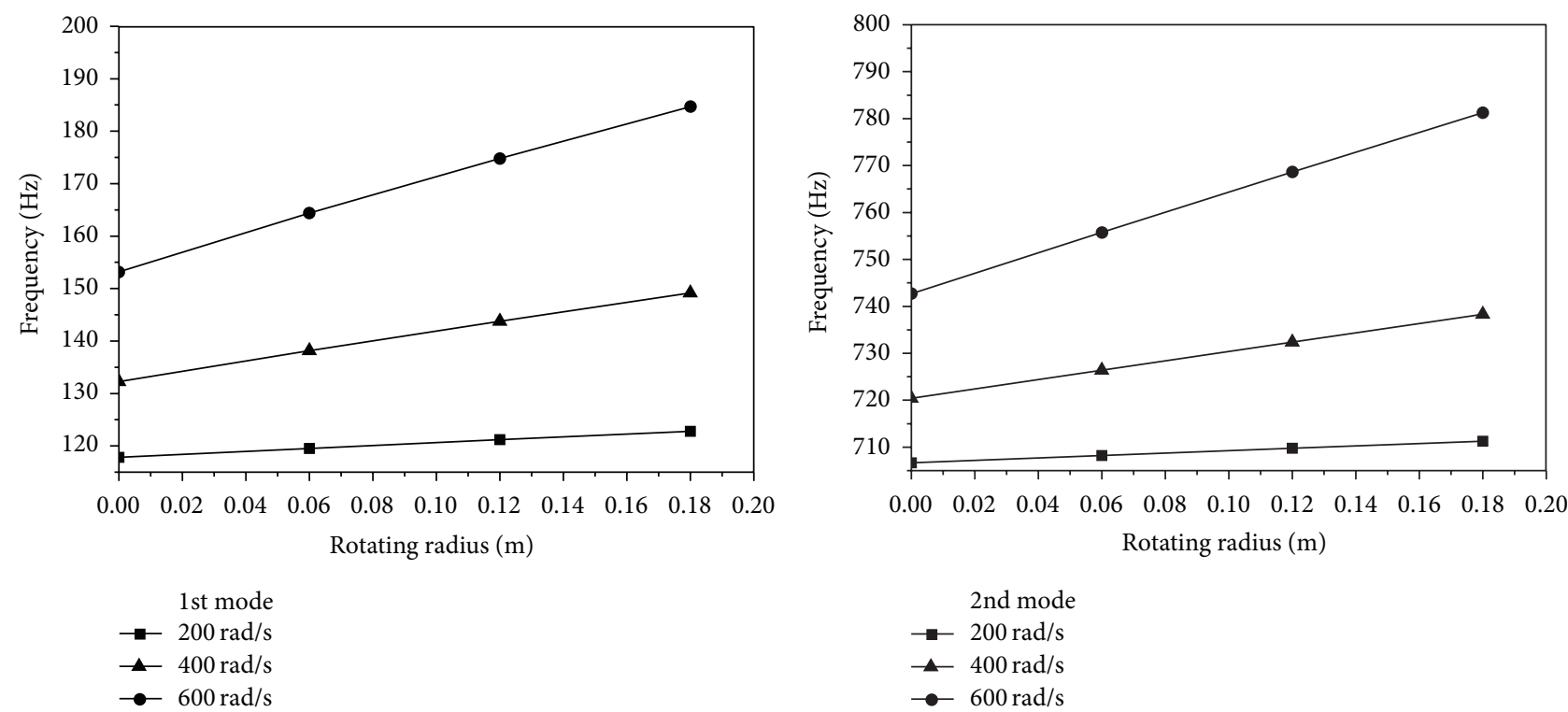

(a) 1st natural frequency
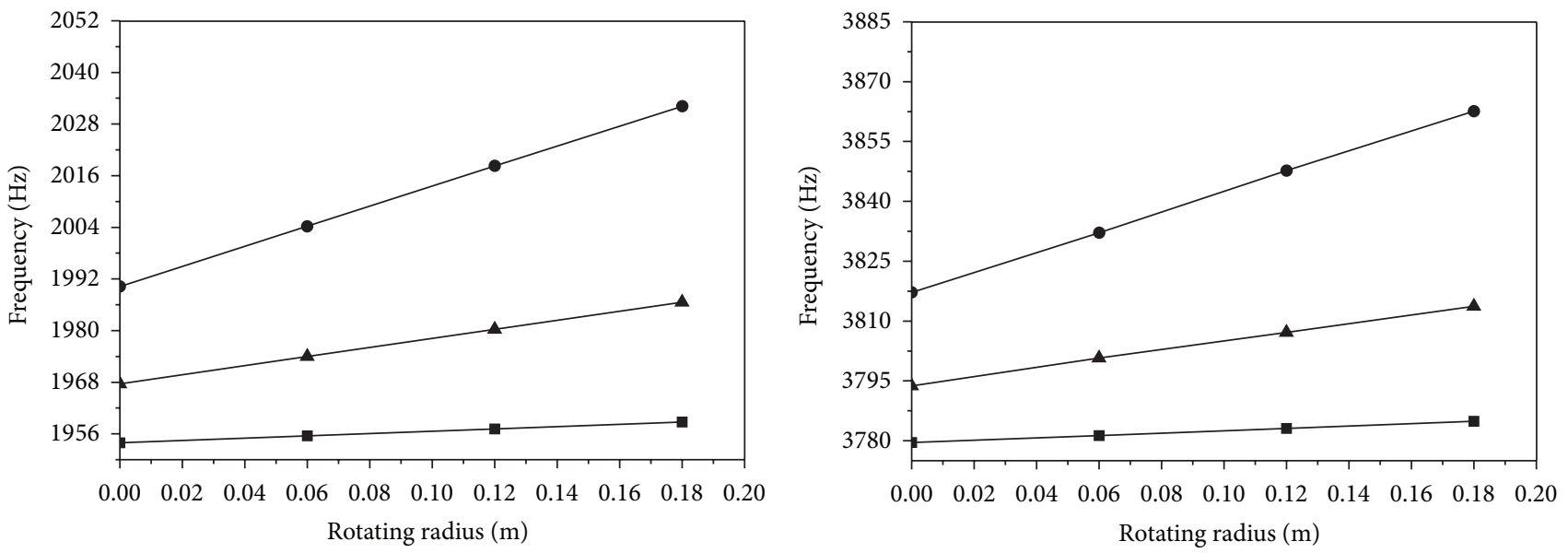

$\begin{aligned} & 3 \mathrm{rd} \mathrm{mode} \\ - & 200 \mathrm{rad} / \mathrm{s} \\ - & 400 \mathrm{rad} / \mathrm{s} \\ - & 600 \mathrm{rad} / \mathrm{s}\end{aligned}$

(c) 3rd natural frequency

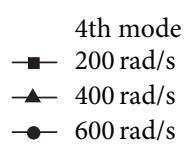

(d) 4 th natural frequency

FIGURE 6: Variations of the frequency with rotating radiuses for different rotating speed.

beam using commercial finite element software are carried out.

\section{Governing Equations}

Figure 1 shows a beam of the length $L$ in Cartesian coordinate with the $x$-axis passing through its centroidal axis. The beam is assumed to be rotating at constant velocity $\Omega$. The axis $z$ is parallel to rotating axis while the axis $y$ is in the plane of rotating. The beam is straight without initial imperfections. Under the action of external load, the deflection of the beam contains two parts: the static transverse deflection $w_{0}$ produced by dead load $p_{0}$ and dynamic deflection $w$ produced by live load $p$. The dead load is constant during the vibration process while the live load is time-related such as aerodynamic load. The dynamic deflection is measured from the reference state. Section rotation $\theta$ contains the effect of shearing strain $\gamma$, and the relation is given by

$$
\gamma=w^{\prime}-\theta
$$

The centrifugal tension $T$ can be expressed as a function of $x$ :

$$
T(x)=\int_{x}^{L} \rho A \Omega^{2}(x+r) d x,
$$




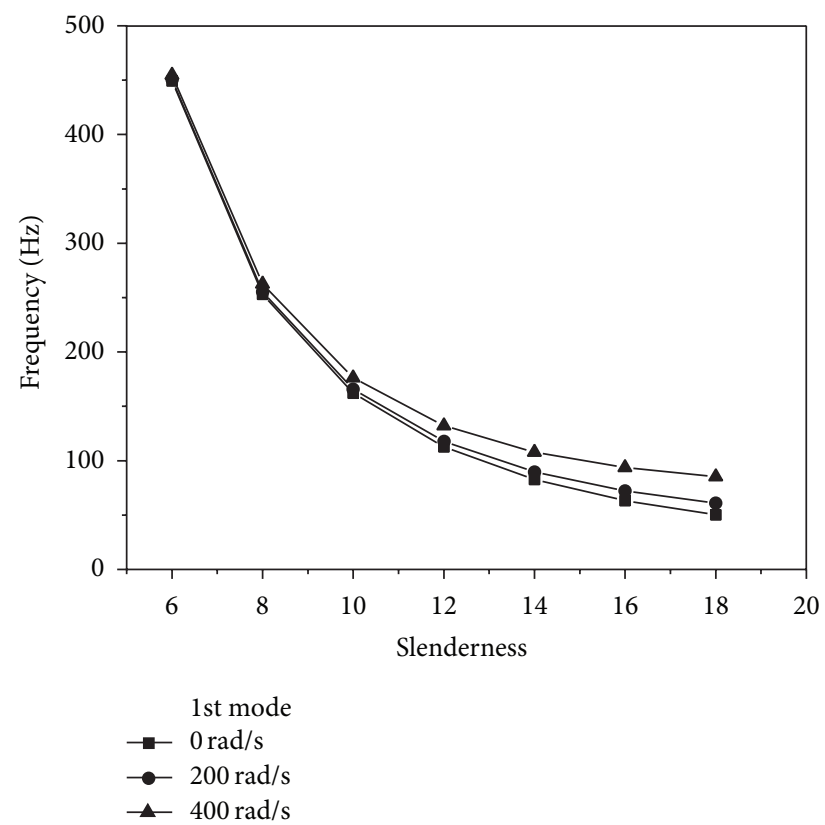

(a) 1st natural frequency

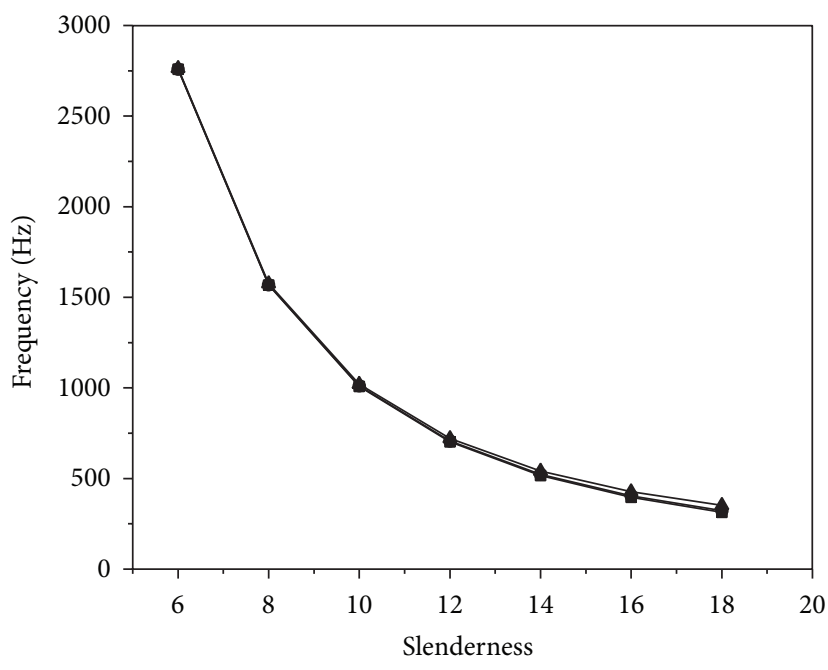

2nd mode

$\rightarrow 0 \mathrm{rad} / \mathrm{s}$

$\rightarrow 200 \mathrm{rad} / \mathrm{s}$

$\neg 400 \mathrm{rad} / \mathrm{s}$

(b) 2nd natural frequency

FIGURE 7: Variations of the frequency with slenderness of beam for different rotating speed.

where $r$ is the rotating radius, $\rho$ is the density, and $A$ is the section area of the beam. The centrifugal tension produces uniform strain $\varepsilon_{0}$ given by

$$
\varepsilon_{0}(x)=\frac{T(x)}{E A} .
$$

The strain-displacement relation for the rotating Timoshenko beams is expressed as

$$
\varepsilon=\varepsilon_{0}-z \theta^{\prime}+\frac{1}{2}\left(w^{\prime}\right)^{2} .
$$

In case of a small deformation assumption, relation (4) can be reduced to a linear form:

$$
\varepsilon=\varepsilon_{0}-z \theta^{\prime}
$$

Then total strain energy $U$ can be written as

$$
U=U_{f}+U_{0}+U_{s}
$$

in which $U_{f}, U_{0}$, and $U_{s}$ denote the live load strain energy, the dead load strain energy, and shear strain energy, respectively. The first two parts of strain energy could be written as the following:

$$
\begin{aligned}
& U_{f}=\frac{1}{2} \iiint \sigma \varepsilon d V, \\
& U_{0}=\iiint \sigma_{0} \varepsilon d V,
\end{aligned}
$$

where $\sigma$ is the bending stress caused by the live load and $\sigma_{0}$ is the conservative initial bending stress caused by the
TABLE 1: Beam dimensions and material properties.

\begin{tabular}{cccccc}
\hline Width & Height & $L$ & $E$ & $\rho$ & $\mu$ \\
\hline $0.02 \mathrm{~m}$ & $0.008 \mathrm{~m}$ & $0.24 \mathrm{~m}$ & $6.89 e 10 \mathrm{~Pa}$ & $2730 \mathrm{~kg} / \mathrm{m}^{3}$ & 0.33 \\
\hline
\end{tabular}

dead load. Since strain caused by the dead load is timeindependent, it makes no difference to the final results and it is left out. Consequently, $\varepsilon$ denotes the normal strain caused by the live load. Nonlinear relation (4) is used to calculate the strain energy $U_{f}$ in (7) for live load and the strain energy $U_{0}$ in (8) for dead load.

The strain $\varepsilon_{0}$ can be expressed in terms of the centrifugal tension. After integrating the expression, a more simplified form of strain energy is obtained. The effect of loads can be introduced through considering strain energy caused by them. In addition, the strain energy due to shear $U_{s}$ is given by

$$
U_{s}=\frac{1}{2} \int_{0}^{L} \kappa A G\left(w^{\prime}-\theta\right)^{2} d x,
$$

where $G$ is the shear modulus and $\kappa$ is the section shape factor which can be expressed as a function of Poisson's ratio. The total strain energy for rotating Timoshenko beams considering a dead load could be presented as the following:

$$
\begin{aligned}
U & =\frac{1}{2}\left[\int _ { 0 } ^ { L } \left\{E I\left(\theta^{\prime}\right)^{2}+T\left(w^{\prime}\right)^{2}+\kappa A G\left(w^{\prime}-\theta\right)^{2}\right.\right. \\
& \left.\left.+\frac{1}{2} E A\left(w^{\prime}\right)^{2}\left(w_{0}^{\prime}\right)^{2}+2 E I w^{\prime \prime} w_{0}^{\prime \prime}\right\} d x\right]+C_{1} .
\end{aligned}
$$



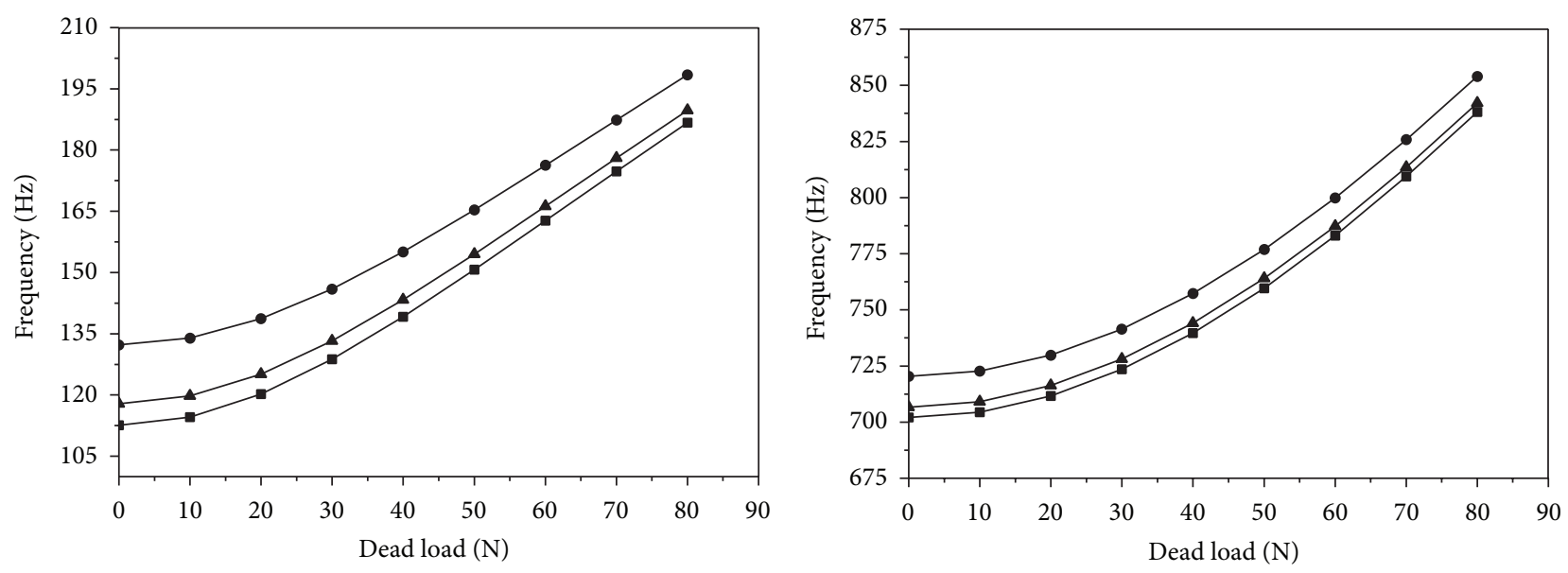

1 st mode

$\rightarrow 0 \mathrm{rad} / \mathrm{s}$

$\neg 200 \mathrm{rad} / \mathrm{s}$

$\rightarrow 400 \mathrm{rad} / \mathrm{s}$

(a) 1st natural frequency
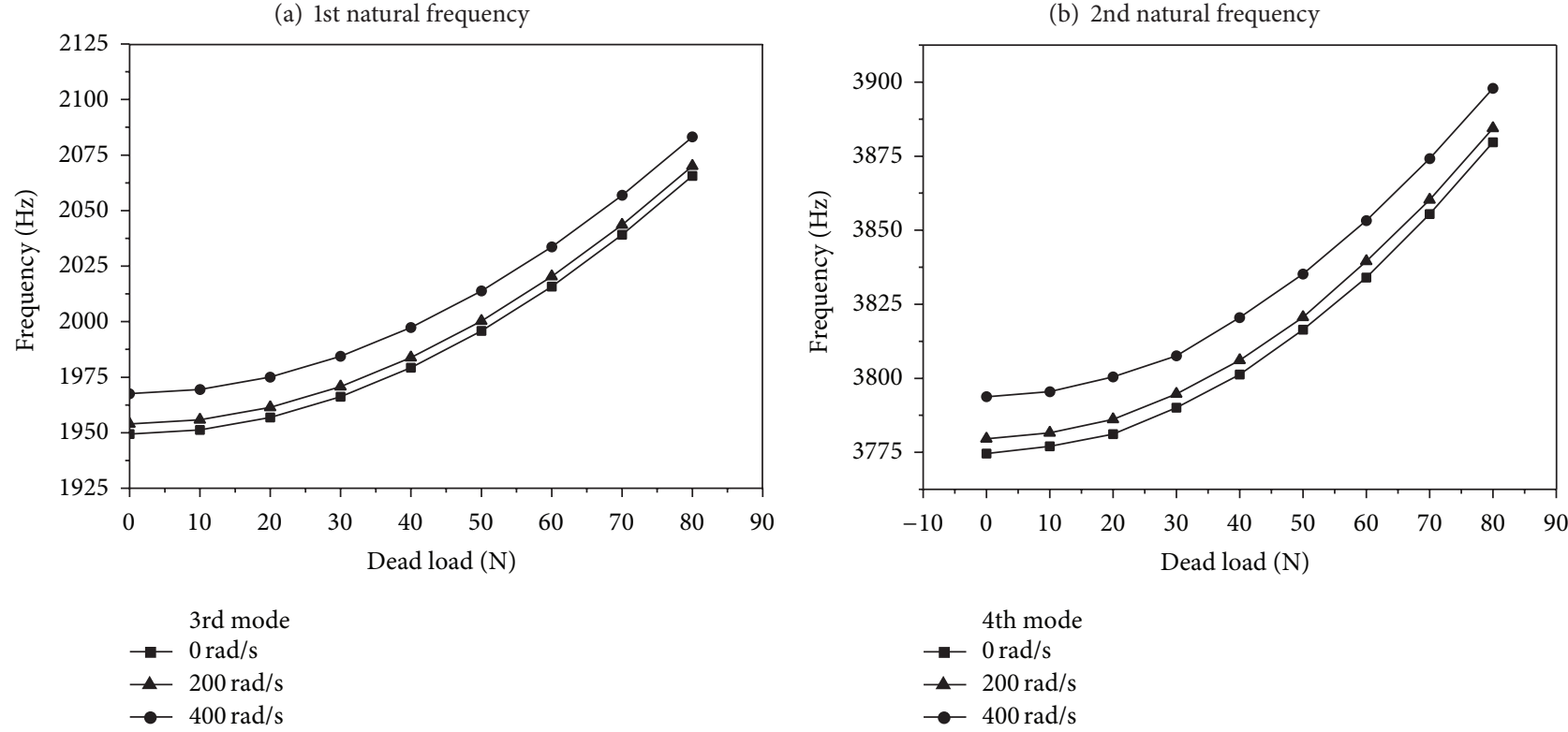

3rd mode

$\rightarrow 0 \mathrm{rad} / \mathrm{s}$

$\neg 200 \mathrm{rad} / \mathrm{s}$

$\longrightarrow 400 \mathrm{rad} / \mathrm{s}$

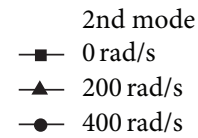

(b) 2nd natural frequency

(c) 3rd natural frequency

(d) 4th natural frequency

FIGURE 8: Variations of the frequency with the dead load for different rotating speed.

The kinetic energy for rotating Timoshenko beams is presented in the form of

$$
T=\frac{1}{2}\left[\int_{0}^{L} \rho I\left(\Omega^{2} \theta^{2}+\dot{\theta}^{2}\right) d x+\int_{0}^{L} \rho A \dot{w}^{2} d x\right]+C_{2}
$$

The variation of the potential energy produced by external load of the beam can be impressed by

$$
\delta W=-\int_{0}^{L}\left(p_{0}+p\right) w d x .
$$

Applying Hamilton's principle to (10) to (12) and taking variations with respect to $w$, the following result is obtained:

$$
\begin{aligned}
& \delta \int_{t_{1}}^{t_{2}}(T-U) d t+\int_{t_{1}}^{t_{2}} \delta W d t=\int_{t_{1}}^{t_{2}} \int_{0}^{L}\left[\left(\rho I\left(\Omega^{2} \theta-\frac{\partial^{2} \theta}{\partial t^{2}}\right)+E I \frac{\partial^{2} \theta}{\partial x^{2}}+k A G\left(\frac{\partial w}{\partial x}-\theta\right)\right) \delta \theta\right. \\
& \left.\quad+\left(p_{0}-\frac{\partial^{2}}{\partial x^{2}}\left(E I \frac{\partial^{2} w_{0}}{\partial x^{2}}\right)\right) \delta w\left(T \frac{\partial^{2} w}{\partial x^{2}}+\kappa A G\left(\frac{\partial^{2} w}{\partial x^{2}}-\frac{\partial \theta}{\partial x}\right)-\rho A \frac{\partial^{2} w}{\partial t^{2}}+\frac{1}{2} E A\left(\frac{\partial w_{0}}{\partial x}\right)^{2} \frac{\partial^{2} w}{\partial x^{2}}-p\right) \delta w\right] d x d t \\
& \quad=0 .
\end{aligned}
$$




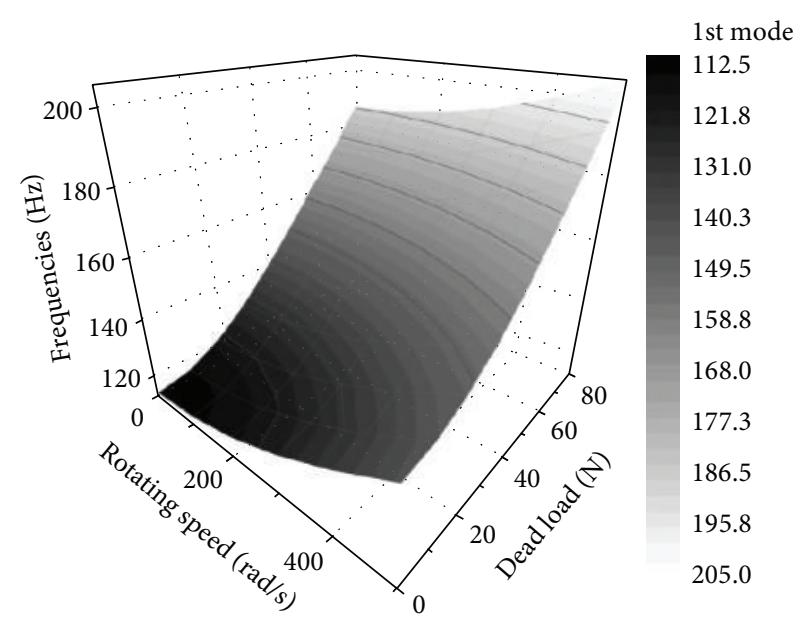

(a) 1st natural frequency

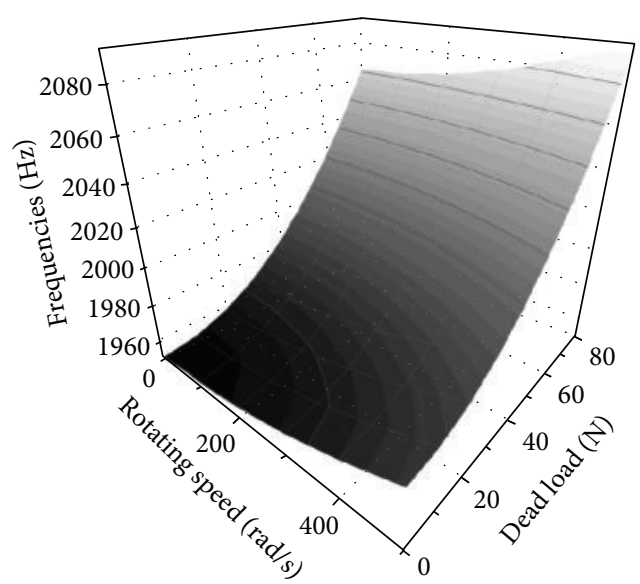

(c) 3rd natural frequency

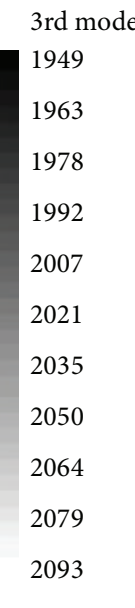

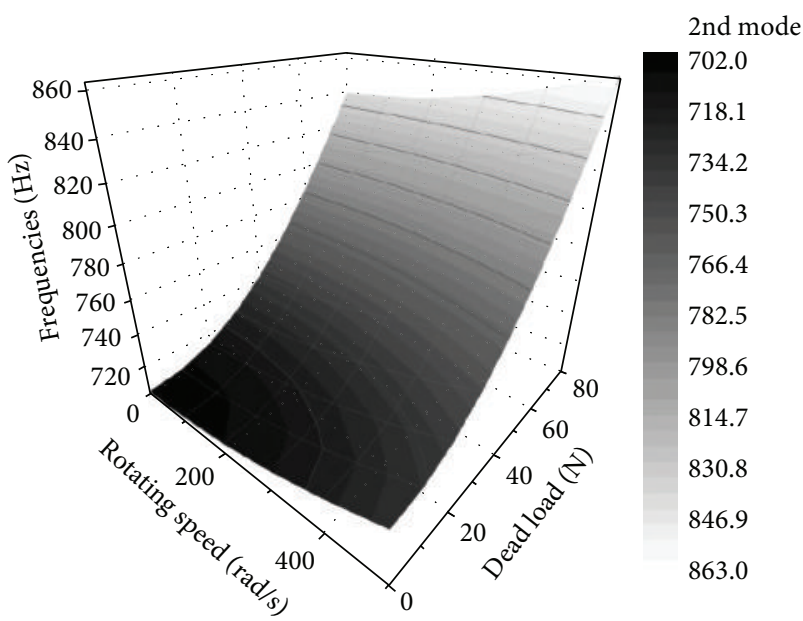

(b) 2nd natural frequency

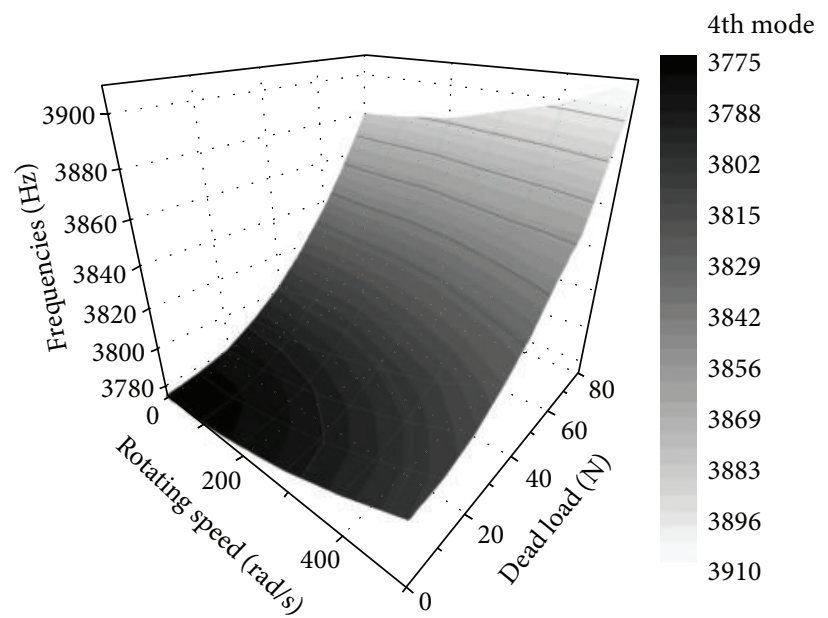

(d) 4th natural frequency

FIGURE 9: Variations of the frequency with both dead load and rotating speed.

In the reference state, the following equilibrium equation exists:

$$
p_{0}-\frac{\partial^{2}}{\partial x^{2}}\left(E I \frac{\partial^{2} w_{0}}{\partial x^{2}}\right)=0
$$

Incorporating (14) into (13), the governing equation for free vibrations of rotating Timoshenko beam with the effect of dead load can be written as

$$
\begin{gathered}
E I \frac{\partial^{2} \theta}{\partial x^{2}}+\kappa A G\left(\frac{\partial w}{\partial x}-\theta\right)+\rho I \Omega^{2} \theta-\rho I \frac{\partial^{2} \theta}{\partial t^{2}}=0 \\
\kappa A G\left(\frac{\partial^{2} w}{\partial x^{2}}-\frac{\partial \theta}{\partial x}\right)+\left(T+\frac{1}{2} E A\left(\frac{\partial w_{0}}{\partial x}\right)^{2}\right) \frac{\partial^{2} w}{\partial x^{2}} \\
-\rho A \frac{\partial^{2} w}{\partial t^{2}}=0 .
\end{gathered}
$$

According to (15b), the following form can be obtained:

$$
\begin{aligned}
& \frac{\partial \theta}{\partial x} \\
& =\frac{\partial^{2} w}{\partial x^{2}} \\
& \quad-\frac{1}{\kappa A G}\left(\rho A \frac{\partial^{2} w}{\partial t^{2}}-\left(T+\frac{1}{2} E A\left(\frac{\partial w_{0}}{\partial x}\right)^{2}\right) \frac{\partial^{2} w}{\partial x^{2}}\right) .
\end{aligned}
$$

Then the following equation can be written by taking the derivative of (15a):

$$
\begin{aligned}
& E I \frac{\partial^{3} \theta}{\partial x^{3}}+\kappa A G\left(\frac{\partial^{2} w}{\partial x^{2}}-\frac{\partial \theta}{\partial x}\right)+\rho I \Omega^{2} \frac{\partial \theta}{\partial x}-\rho I \frac{\partial^{3} \theta}{\partial t^{2} \partial x} \\
& \quad=0 .
\end{aligned}
$$

Taking (16) into (17), the final governing equations can be uncoupled to produce a fourth-order equation in $w$. 
Considering the axial force and initial deformation, the partial differential equation can be written as

$$
\begin{aligned}
E I \frac{\partial^{4} w}{\partial x^{4}} & -\frac{\rho I E}{\kappa G} \frac{\partial^{4} w}{\partial t^{2} \partial x^{2}} \\
+ & \frac{E I}{\kappa A G}\left(T+\frac{1}{2} E A\left(\frac{\partial w_{0}}{\partial x}\right)^{2}\right) \frac{\partial^{4} w}{\partial x^{4}}+\rho A \frac{\partial^{2} w}{\partial t^{2}} \\
& -\left(T+\frac{1}{2} E A\left(\frac{\partial w_{0}}{\partial x}\right)^{2}\right) \frac{\partial^{2} w}{\partial x^{2}}+\rho I \Omega^{2} \frac{\partial^{2} w}{\partial x^{2}} \\
& -\frac{\rho^{2} I \Omega^{2}}{\kappa G} \frac{\partial^{2} w}{\partial t^{2}} \\
+ & \frac{\rho I \Omega^{2}}{\kappa A G}\left(T+\frac{1}{2} E A\left(\frac{\partial w_{0}}{\partial x}\right)^{2}\right) \frac{\partial^{2} w}{\partial x^{2}}-\rho I \frac{\partial^{4} w}{\partial x^{2} \partial t^{2}} \\
+ & \frac{\rho^{2} I}{\kappa G} \frac{\partial^{4} w}{\partial t^{4}} \\
& -\frac{\rho I}{\kappa A G}\left(T+\frac{1}{2} E A\left(\frac{\partial w_{0}}{\partial x}\right)^{2}\right) \frac{\partial^{4} w}{\partial x^{2} \partial t^{2}}=0 .
\end{aligned}
$$

Equation (18) is solved by means of the Galerkin method. The deflection is assumed to be

$$
w(y, t)=\sum_{i=1}^{n} \phi_{i}(y) q_{i}(t) .
$$

Governing equation (18) can be represented as

$$
D \dddot{q}+M \ddot{q}+K q=0,
$$

\begin{tabular}{|c|c|c|c|}
\hline Dead load & 1st mode & 2nd mode & 3rd mode \\
\hline \multicolumn{4}{|l|}{$0 \mathrm{~N}$} \\
\hline Test & 115.56 & 700.46 & 1907.91 \\
\hline Present theory & 112.71 & 706.36 & 1977.84 \\
\hline Error (\%) & 2.53 & 0.84 & 3.54 \\
\hline \multicolumn{4}{|l|}{$10 \mathrm{~N}$} \\
\hline Test & 116.30 & 702.53 & 1911.46 \\
\hline Present theory & 114.70 & 708.80 & 1979.73 \\
\hline Error (\%) & 1.39 & 0.88 & 3.45 \\
\hline \multicolumn{4}{|l|}{$15 \mathrm{~N}$} \\
\hline Test & 118.82 & 703.40 & 1912.67 \\
\hline Present theory & 117.10 & 711.83 & 1982.09 \\
\hline Error (\%) & 1.47 & 1.18 & 3.50 \\
\hline \multicolumn{4}{|l|}{$20 \mathrm{~N}$} \\
\hline Test & 120.17 & 704.51 & 1917.10 \\
\hline Present theory & 120.33 & 716.05 & 1985.39 \\
\hline Error (\%) & 0.13 & 1.61 & 3.44 \\
\hline \multicolumn{4}{|l|}{$25 \mathrm{~N}$} \\
\hline Test & 121.93 & 706.46 & 1923.12 \\
\hline Present theory & 124.28 & 721.42 & 1989.62 \\
\hline Error (\%) & 1.89 & 2.07 & 3.34 \\
\hline \multicolumn{4}{|l|}{$30 \mathrm{~N}$} \\
\hline Test & 122.97 & 708.32 & 1927.85 \\
\hline Present theory & 128.83 & 727.90 & 1994.77 \\
\hline Error (\%) & 4.55 & 2.69 & 3.35 \\
\hline \multicolumn{4}{|l|}{$35 \mathrm{~N}$} \\
\hline Test & 127.54 & 710.60 & 1933.23 \\
\hline Present theory & 133.87 & 735.47 & 2000.84 \\
\hline Error (\%) & 4.73 & 3.38 & 3.38 \\
\hline \multicolumn{4}{|l|}{$40 \mathrm{~N}$} \\
\hline Test & 133.08 & 714.68 & 1944.04 \\
\hline Present theory & 139.27 & 744.06 & 2007.82 \\
\hline Error (\%) & 4.44 & 3.95 & 3.18 \\
\hline
\end{tabular}

where

$$
\begin{aligned}
D_{i j} & =\int_{0}^{L} \frac{\rho^{2} I}{\kappa G} \phi_{i} \phi_{j} d x, \\
M_{i j} & =\int_{0}^{L} \rho I\left(1+\frac{E}{\kappa G}\right. \\
& \left.+\frac{1}{\kappa A G}\left(T+\frac{1}{2} E A\left(\frac{\partial w_{0}}{\partial x}\right)^{2}\right)\right) \phi_{i}^{\prime} \phi_{j}^{\prime} d x \\
& +\int_{0}^{L} \rho A\left(1-\frac{\rho I \Omega^{2}}{\kappa A G}\right) \phi_{i} \phi_{j} d x, \\
K_{i j} & =\int_{0}^{L} E I\left(1+\frac{1}{\kappa A G}\left(T+\frac{1}{2} E A\left(\frac{\partial w_{0}}{\partial x}\right)^{2}\right)\right) \\
& \cdot \phi_{i}^{\prime \prime} \phi_{j}^{\prime \prime} d x+\int_{0}^{L}\left(T+\frac{1}{2} E A\left(\frac{\partial w_{0}}{\partial x}\right)^{2}\right. \\
& \left.-\rho I \Omega^{2}\left(1+\frac{1}{\kappa A G}\left(T+\frac{1}{2} E A\left(\frac{\partial w_{0}}{\partial x}\right)^{2}\right)\right)\right) \\
& \cdot \phi_{i}^{\prime} \phi_{j}^{\prime} d x .
\end{aligned}
$$

The above formulation in which terms including centrifugal force $T$ denote the effect of rotation while terms including
TABLE 2: Comparison of the first three natural frequencies under different dead loads.

initial deformation $w_{0}$ denote the effect of dead load is used and how these loads impact dynamic beams is discussed from results of numerical computations.

\section{Experimental Verification}

To verify the result of the natural frequencies of the beam with a dead load, the modal test with the dead load is carried out using LMS TEST.LAB. The main aim is to investigate the effect of dead load on dynamic characteristic and our device requires a fixed support. On this occasion, the effect of rotation is verified through numerical method. Rotating effect is left out during the test. Experimental setup is shown in Figure 2.

The test specimen is an aluminum alloy beam as shown in Figure 3. The geometric dimension and material property are illustrated in Table 1. Two holes are drilled at one rim of the beam to achieve the clamped boundary condition with 
TABLE 3: Change rates of natural frequencies under the effect of rotating speed or dead load.

\begin{tabular}{|c|c|c|c|c|}
\hline$\omega_{x} / \omega_{0}$ & 1st mode & 2nd mode & 3rd mode & 4 th mode \\
\hline With rotating speed, no dead load & 1.17467 & 1.02615 & 1.00937 & 1.00508 \\
\hline No rotating speed, with dead load, & 1.23577 & 1.05357 & 1.01532 & 1.00708 \\
\hline With rotating speed and dead load & 1.37714 & 1.07864 & 1.02459 & 1.01216 \\
\hline
\end{tabular}

TABLE 4: Comparison of the first four natural frequencies with different rotating speed.

\begin{tabular}{lccccc}
\hline Rotating speed & Beam theory numerical & 1st mode & 2nd mode & 3rd mode & 4th mode \\
\hline \multirow{2}{*}{$0 \mathrm{rad} / \mathrm{s}$} & Timoshenko (error) & $112.61(0.02 \%)$ & $702.03(0.12 \%)$ & $1949.37(0.29 \%)$ & $3774.58(0.54 \%)$ \\
& Euler (error) & $112.71(0.07 \%)$ & $706.36(0.49 \%)$ & $1977.84(1.16 \%)$ & $3876.00(2.13 \%)$ \\
& FEM & 112.63 & 702.89 & 1955.13 & 3795.08 \\
\hline \multirow{2}{*}{$200 \mathrm{rad} / \mathrm{s}$} & Timoshenko (error) & $117.85(0.02 \%)$ & $706.67(0.13 \%)$ & $1953.95(0.30 \%)$ & $3779.57(0.53 \%)$ \\
& Euler (error) & $117.95(0.07 \%)$ & $710.99(0.49 \%)$ & $1982.40(1.16 \%)$ & $3880.70(2.13 \%)$ \\
& FEM & 117.87 & 707.54 & 1959.74 & 3799.88 \\
\hline \multirow{2}{*}{$400 \mathrm{rad} / \mathrm{s}$} & Timoshenko (error) & $132.28(0.01 \%)$ & $720.39(0.13 \%)$ & $1967.64(0.30 \%)$ & $3793.75(0.54 \%)$ \\
& Euler (error) & $132.37(0.06 \%)$ & $724.71(0.47 \%)$ & $1996.03(1.14 \%)$ & $3894.78(2.11 \%)$ \\
& FEM & 132.29 & 721.30 & 3814.23 \\
\hline
\end{tabular}

bolts. Eight points are uniformly distributed as modal test excitation points. Single-input single-output hammer (PCB hammer 086CD4) based impact is used as the modal test method. The eight test points are impacted by the hammer in sequence. An accelerometer (PCB 333B32) is located at point number 2 to receive the acceleration information. Foundation and fixtures are shown in Figure 4.

To apply a dead load on the beam, a method which is easy to implement is proposed as shown in Figure 5 based on the following consideration. The device used to apply the dead load ought to solve two difficult points when the beam is in vibration environments. Firstly, the loading equipment should not produce added mass to specimen. Another point is to keep the stability of the dead load when the beam is under dynamic load. Finally, an elastic string of high tensile strength is selected and a bearing rod with small quality is set at the end of beam.

The elastic string is stretched to a calculated length and fixed at the designed positions. The top of the bearing bar is just pressed by the string and the tension of the elastic string is converted to the dead load on the beam. By changing the length of the string, the dead load with different value could be controlled.

The lightest wood, Balsa Wood, is chosen to make the bearing bar in order to reduce the effect of additional mass on the beam. The stiffness of elastic string is small enough to ensure that the variation of the tensile force caused by the beam vibration is negligible. Through this way, the dead load could remain relatively steady. According to the range of acceleration sensor, the first three natural frequencies are measured from impact test as shown in Table 2.

Timoshenko beam governing equation (20) can be simplified into Euler-Bernoulli model for the theoretical calculation in Table 2. Natural frequencies are obtained by solving the corresponding eigenvalue problem of governing equation. The modal test results agree well with the theoretical results and indicate increasing with the dead load.

\section{Numerical Verification}

Data in Table 1 is used while conducting numerical verification. Table 3 shows the natural frequencies under rotating speed $400 \mathrm{rad} / \mathrm{s}$ or dead load $40 \mathrm{~N}$, in which $\omega_{0}$ is the natural frequency without rotating speed or dead load, and $\omega_{x}$ is the natural frequency with extra stress caused by external force existing. The effect of rotation and dead load is more obvious in the lower-order frequencies.

The first four natural frequencies of rotating Timoshenko beams without dead loads are calculated through the commercial analysis software Nastran, in which the beam is meshed into 100 beam elements here. Different rotating speeds are transformed as initial internal force to modal analysis. To identify the accuracy of theoretical modal, Table 4 gives a comparison of the first four natural frequencies between Timoshenko/Euler beam theory and finite element method without the dead load. The natural frequencies of rotating Euler beam are derived from the simplified equation ignoring shear deformation and rotary inertia without dead load. Both theoretical results are compared with the finite element result, and it shows that result obtained from governing equation for Timoshenko beams agrees better with the FEM simulation result. The percentage error for Euler beams is three times larger than that of Timoshenko beams and becomes larger in high-order frequencies. Variation shows that natural frequencies increase more and more rapidly with the rotating speed.

\section{Result and Discussion}

Effect of some other factors on the natural frequency of the beam is studied. Figure 6 shows the variation of the first four natural frequencies with rotating radius $r$ for different rotating speeds. The relation of natural frequencies with rotating radius is approximately linearity and gradient is larger when rotating speed is higher. 


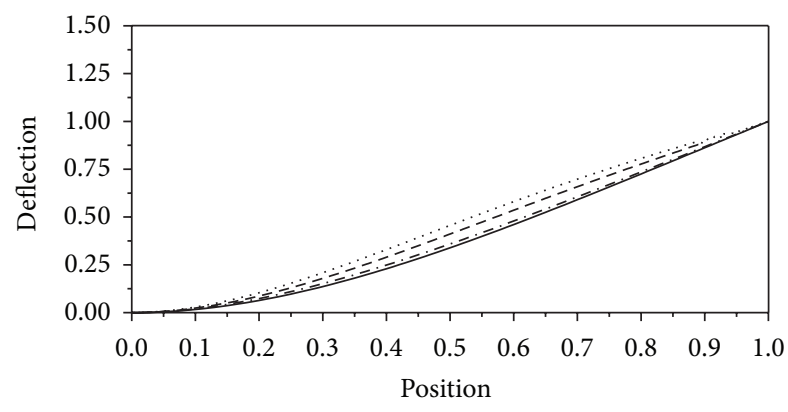

1st mode

_ No rotating speed, no dead load

-..- With rotating speed, no dead load

- - - No rotating speed, with dead load

With rotating speed, with dead load

(a) 1st mode shape

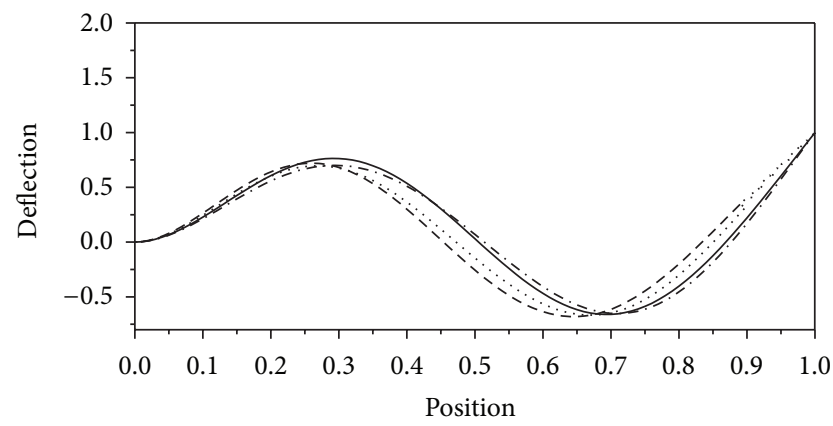

3rd mode

_ No rotating speed, no dead load

-.. - With rotating speed, no dead load

- - - No rotating speed, with dead load

..... With rotating speed, with dead load

(c) 3rd mode shape

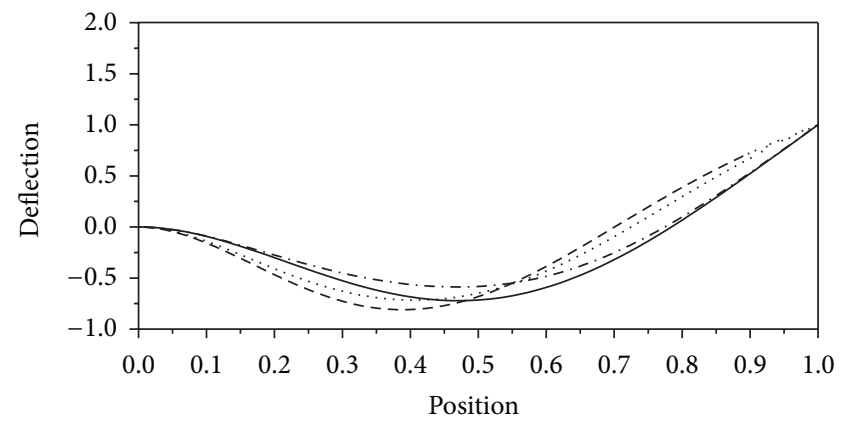

2nd mode

- No rotating speed, no dead load

...- With rotating speed, no dead load

- - - No rotating speed, with dead load

With rotating speed, with dead load

(b) 2nd mode shape

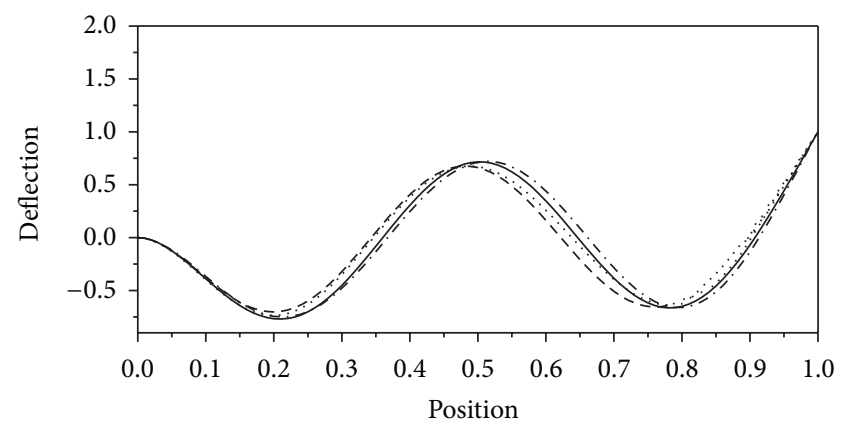

4th mode

_ No rotating speed, no dead load

..- With rotating speed, no dead load

- - - No rotating speed, with dead load

..... With rotating speed, with dead load

(d) 4th mode shape

FIgURE 10: Mode shape variations due to the rotation and the dead load.

The natural frequency is also determined for different slenderness under incremental rotating speeds and the variation trend is shown in Figure 7. The natural frequency drops sharply with the increase of slenderness for a thicker beam and trends to be flattened when the beam gets thinner. Then the effect of rotation becomes distinct. For higher-order modes, greater rotating speed is required to cancel out the effect of size.

Considering the effect of dead load, first four natural frequencies are calculated for different rotating speeds as shown in Figure 8. It is clear that the natural frequency of the beam increases faster with the dead load rising. As shown in Figure 8(a), growth for the first mode frequency tends to be stable when the dead load is about over $40 \mathrm{~N}$. This leads to a similar linear relationship between natural frequency and dead load. Figures 8(b), 8(c), and 8(d) show, however, the variations of the natural frequencies of the other three orders, in which the growth rates of frequencies keep increasing with the dead load.

When both dead load and centrifugal tension exist concurrently, natural frequencies show a more obvious growth since both factors have a positive effect on natural frequencies as shown in Figure 9.

For a rotating beam, especially under a dead load, it is useful to obtain the mode shape for design. The first four modes are shown in Figure 10. There areobvious differences among the four curves.

\section{Conclusion}

The effect of the rotation and the dead load on Timoshenko beams has been studied. First, considering the predeformation caused by both the rotating speed and the dead load, governing formation of cantilever beam is derived and the natural vibration is obtained. Next, the effect of rotation on beams is verified by acquiring natural frequencies using a commercial finite element code, and a suit of modal experiment for cantilever beam is designed to verify the effect of the dead load. Comparing theoretical result with the numerical or experimental results, there is a good agreement with each other. Finally, the mixing effect of both the rotation 
and the dead load on natural frequencies and modes is demonstrated and some conclusions are drawn.

The present study shows that the natural frequencies increase gradually when dead load is larger because of resulting stiffness variation. Similarly, the natural frequencies grow when rotating speed is raised. The natural frequencies appear more obviously increasing while both the rotation and the dead load exist. The two factors also impact the mode shapes of beam. In addition, Timoshenko beam model shows higher accuracy since transverse shear effect is considered. The present work also hints that the effect of both the gas pressure and rotating has to be introduced in numerical simulation of dynamical response for rotating blade.

\section{Conflict of Interests}

The authors declare that there is no conflict of interests regarding the publication of this paper.

\section{Acknowledgments}

This work is supported by the National Basic Research Program of China [no. 2013CB035704]; the National Nature Science Foundation of China [no. 11472206]; Hong Kong RGC-GRF Grant [Project no. CityU 118212], and Strategic Research Grant, City University of Hong Kong [Project no. CityU-SRG 7004176].

\section{References}

[1] T. Yokoyama, "Free vibration characteristics of rotating Timoshenko beams," International Journal of Mechanical Sciences, vol. 30, no. 10, pp. 743-755, 1988.

[2] H. Du, M. K. Lim, and K. M. Liew, "Power series solution for vibration of a rotating Timoshenko beam," Journal of Sound and Vibration, vol. 175, no. 4, pp. 505-523, 1994.

[3] S. C. Lin and K. M. Hsiao, "Vibration analysis of a rotating Timoshenko beam," Journal of Sound and Vibration, vol. 240, no. 2, pp. 303-322, 2001.

[4] S. S. Rao and R. S. Gupta, "Finite element vibration analysis of rotating timoshenko beams," Journal of Sound and Vibration, vol. 242, no. 1, pp. 103-124, 2001.

[5] J. R. Banerjee and A. J. Sobey, "Energy expressions for rotating tapered Timoshenko beams," Journal of Sound and Vibration, vol. 254, no. 4, pp. 818-822, 2002.

[6] J. R. Banerjee, H. Su, and D. R. Jackson, "Free vibration of rotating tapered beams using the dynamic stiffness method," Journal of Sound and Vibration, vol. 298, no. 4-5, pp. 1034-1054, 2006.

[7] O. O. Ozgumus and M. O. Kaya, "Vibration analysis of a rotating tapered Timoshenko beam using DTM," Meccanica, vol. 45, no. 1, pp. 33-42, 2010.

[8] J. M. Kelly, J. L. Sackman, and A. Javid, "The influence of preform on the modes of vibrating beams," Earthquake Engineering and Structural Dynamics, vol. 20, no. 12, pp. 1145-1157, 1991.

[9] H. Takabatake, "Effects of dead loads in static beams," Journal of Structural Engineering, vol. 116, no. 4, pp. 1102-1120, 1990.

[10] H. Takabatake, "Effect of dead loads on natural frequencies of beams," Journal of Structural Engineering, vol. 117, no. 4, pp. 1039-1052, 1991.
[11] J. Zhang and W.-X. Ren, "A dynamic stiffness method for determining natural frequencies of beams with allowance for dead load," International Journal of Structural Stability and Dynamics, vol. 9, no. 4, pp. 777-790, 2009.

[12] S.-J. Zhou and X. Zhu, "Analysis of effect of dead loads on natural frequencies of beams using finite-element techniques," Journal of Structural Engineering, vol. 122, no. 5, pp. 512-516, 1996. 


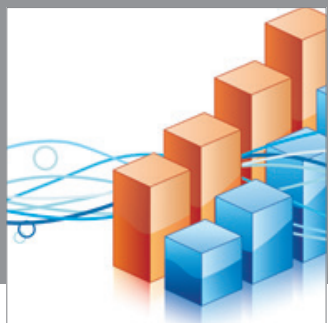

Advances in

Operations Research

mansans

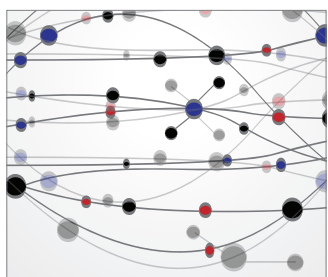

The Scientific World Journal
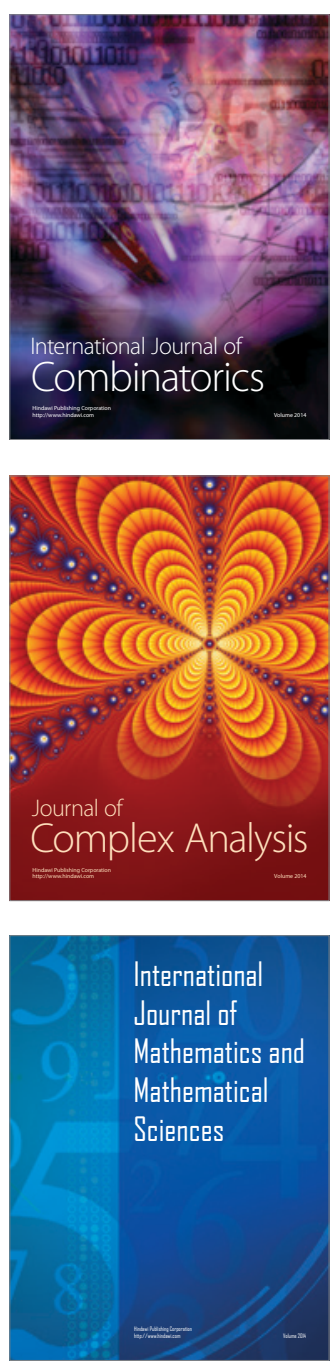
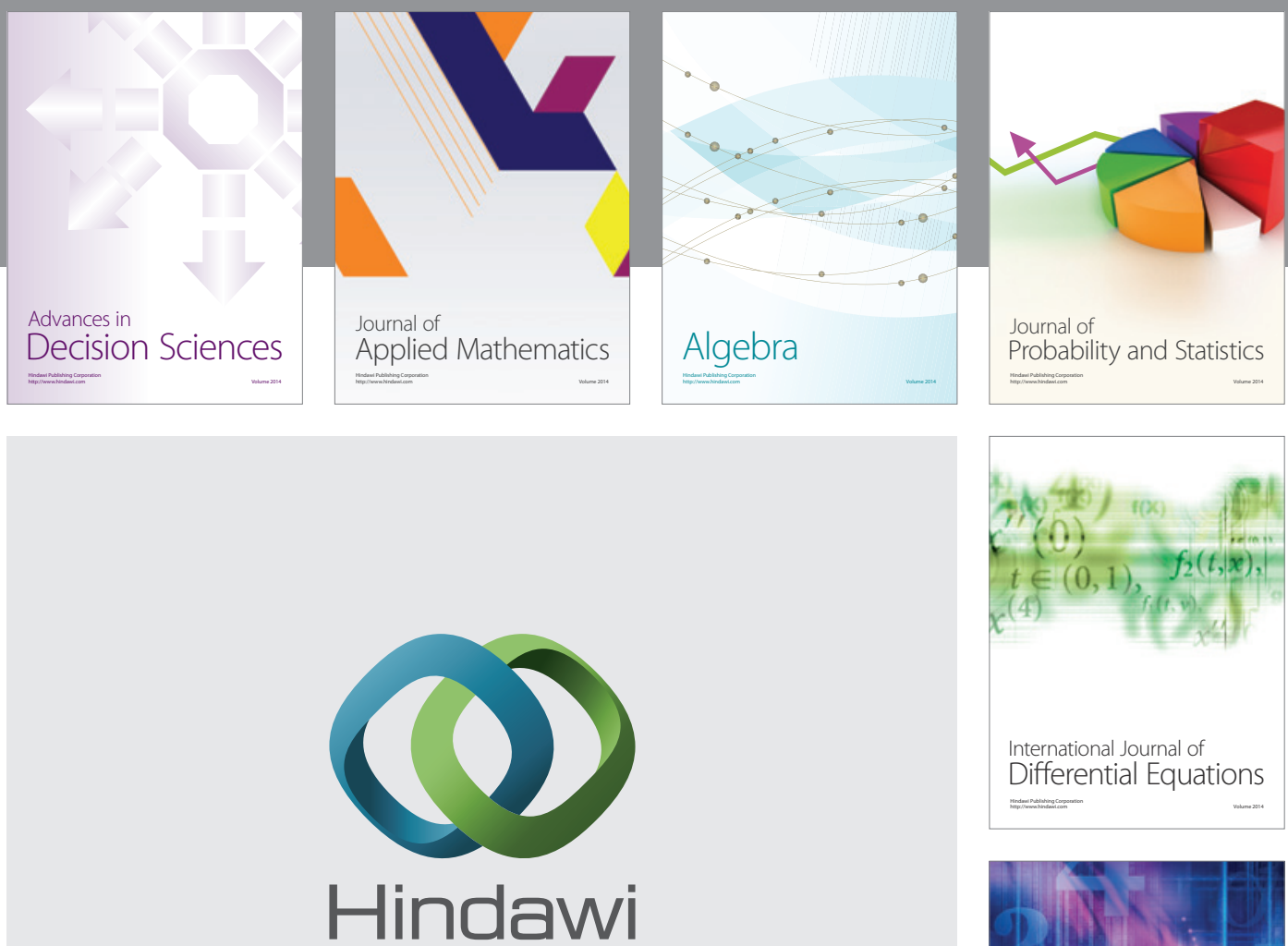

Submit your manuscripts at http://www.hindawi.com
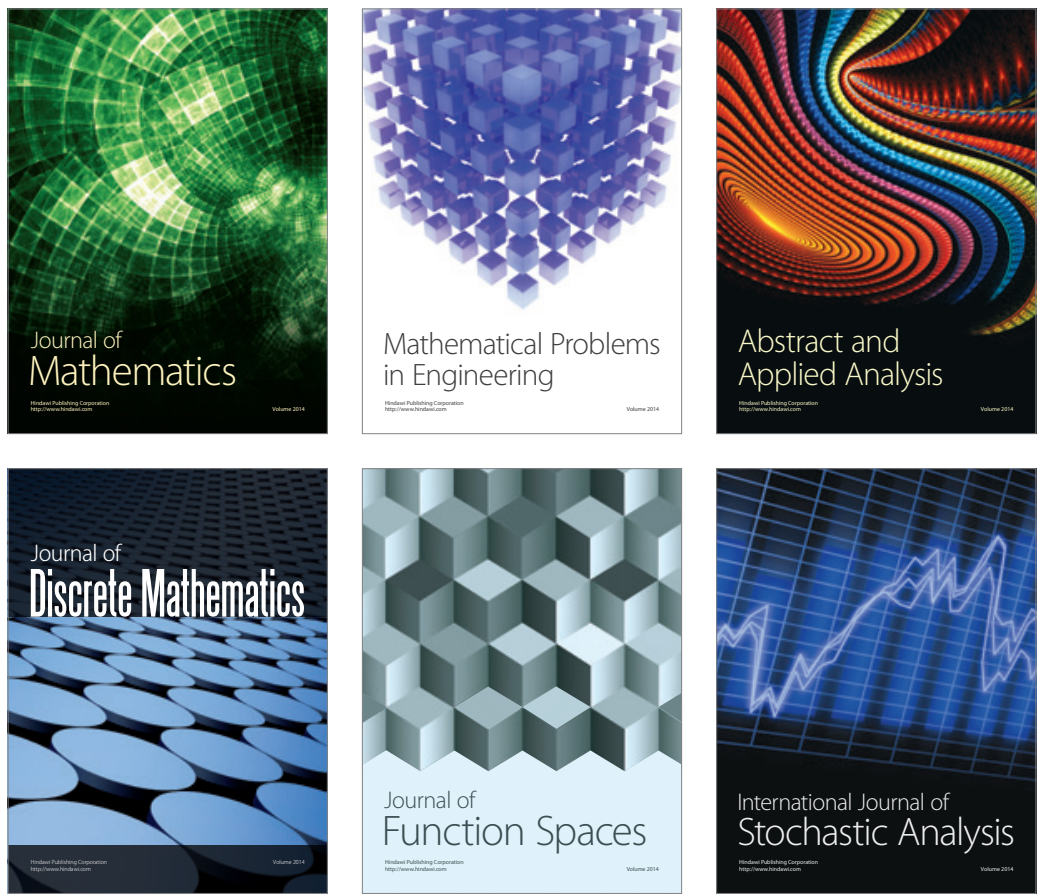

Journal of

Function Spaces

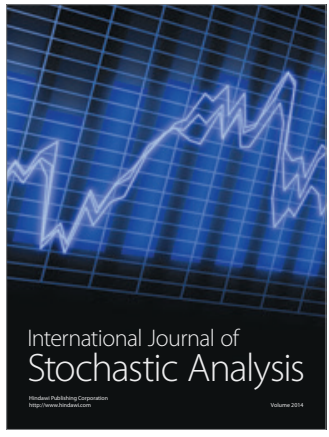

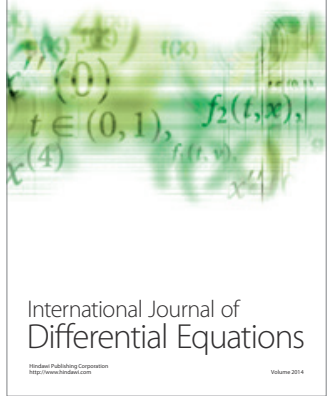
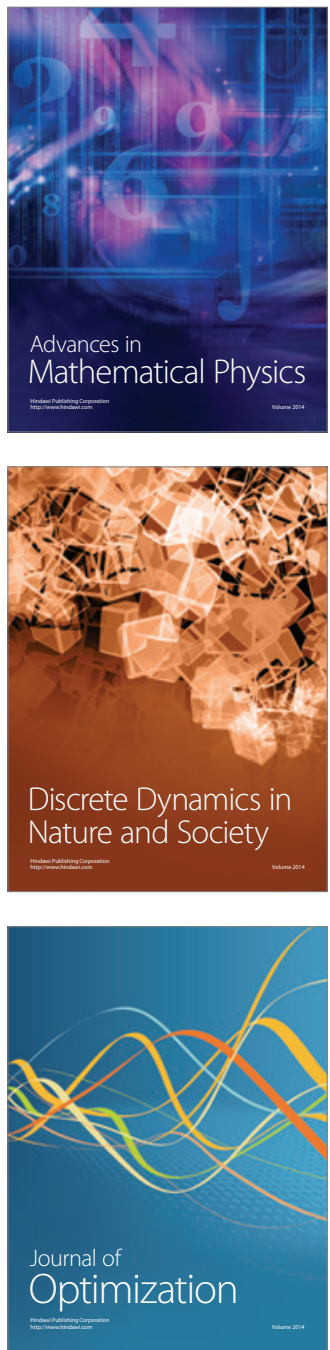